\title{
Generation of high-quality electron beams by ionization injection in a single acceleration stage
}

\author{
Nasr A.M. Hafz, Song Li, Guangyu Li, Mohammad Mirzaie, Ming Zeng, and Jie Zhang \\ Key Laboratory for Laser Plasmas (MOE) and Department of Physics and Astronomy, Collaborative Innovation Center of IFSA, \\ Shanghai Jiao Tong University, Shanghai 200240, China \\ (Received 11 April 2016; revised 12 June 2016; accepted 12 July 2016)
}

\begin{abstract}
Ionization-induced electron injection in laser wakefield accelerators, which was recently proposed to lower the laser intensity threshold for electron trapping into the wake wave, has the drawback of generating electron beams with large and continuous energy spreads, severely limiting their future applications. Complex target designs based on separating the electron trapping and acceleration stages were proposed as the only way for getting small energy-spread electron beams. Here, based on the self-truncated ionization-injection concept which requires the use of unmatched laser-plasma parameters and by using tens of TW laser pulses focused onto a gas jet of helium mixed with low concentrations of nitrogen, we demonstrate single-stage laser wakefield acceleration of multi-hundred $\mathrm{MeV}$ electron bunches with energy spreads of a few percent. The experimental results are verified by PIC simulations.
\end{abstract}

Keywords: ionization injection; laser-plasma acceleration; PIC simulations; self-truncation; wakefield

\section{Introduction}

Since it was proposed in $1979^{[1]}$, laser wakefield acceleration (LWFA) has attracted a lot of attention of many groups around the world. Attributed to its ultra-high acceleration gradients $\left(\sim 1 \mathrm{GV} \mathrm{cm}^{-1}\right)$, the LWFA scheme might be considered as a basis for future compact electronpositron colliders ${ }^{[2-5]}$ and ultra-compact $\mathrm{x}$-ray free-electron lasers $^{[6,7]}$. The LWFA research has achieved a significant breakthrough in $2004^{[8-10]}$ when self-injected quasimonoenergetic electron beams were firstly obtained in experiments under the highly nonlinear 'bubble' regime ${ }^{[11,12]}$. In this regime, electrons of an underdense plasma produced by pure hydrogen or helium gas jets, are expelled radially by the ponderomotive force of a focused ultra-intense laser pulse, leading to form a nearly spherical ionic cavity (bubble) whose size corresponds to the plasma wavelength $\lambda_{p}$. The bubble propagates together with the laser pulse at a velocity close to the speed of light $c$, and experiences evolution due to the evolution of the laser pulse during its propagation in the plasma. During the variation of bubble size an electron density spike builds up at its back and eventually breaks, causing electron injection ${ }^{[13-15]}$ inside the

Correspondence to: N. A. M. Hafz, Key Laboratory for Laser Plasmas (MOE) and Department of Physics and Astronomy, Collaborative Innovation Center of IFSA, Shanghai Jiao Tong University, 800 Dongchaun Road, Minhang District, Shanghai 200240, China. Email: nasr@ sjtu.edu.cn. bubble. The self-injected electrons witness the longitudinal accelerating field inside the bubble and are accelerated with low energy spread. After 2004, dramatic progress has been made in the LWFA research ${ }^{[2,16-18]}$, including the latest experimental results by Leemans et al. ${ }^{[19]}$ on the generation of self-injected monoenergetic electrons with energy up to 4.2 GeV, from 9-cm-long capillary discharge waveguide powered by laser pulses with peak power up to $300 \mathrm{TW}$. However, experimental studies ${ }^{[20]}$ have shown that the selfinjection process is highly nonlinear and uncontrollable as it requires large laser normalized vector potential $a_{0}$, to trigger the injection in single low- $Z$ gas plasma, where $a_{0}=\sqrt{\left(P(\mathrm{TW}) \times 10^{3}\right) / 21.5} \times \lambda_{0}(\mu \mathrm{m}) / w_{0}(\mu \mathrm{m}), P$ is the laser power, $\lambda_{0}$ and $w_{0}$ are the laser's wavelength and focal spot size.

Recently, a novel electron injection scheme based on ionization ${ }^{[21-24]}$ was proposed in order to reduce the laser intensity threshold for electron trapping. This scheme utilizes the high ionization potential of the inner-shell electrons relative to outer-shell electrons of a high- $Z$ doped gas (such as nitrogen) mixed with the usual low- $Z$ host gas (helium or hydrogen) in order to control the initial injection phases of the ionized inner-shell electrons. This regime has been demonstrated in a few experiments ${ }^{[23,24]}$; however, electron beams produced by it usually had very large energy spreads reaching 60\%-100\%, limiting the applications of those beams. In order to overcome the continuous 
injection of electrons, a two-stage accelerating configuration was recently proposed ${ }^{[25,26]}$ as the way to achieve small energy-spread (high-quality) electron beams by ionization injection. In such scheme, the injection and acceleration stages were separated and manipulated in two different gas cells or gas jets. However, a single-stage ionization-injection scheme which can overcome the problem of continuous injection (from the doped gas thus generating high-quality electron beams) must be favored for its simple implementation in experiments and applications. The feasibility of a single-stage, self-truncated ionization-injection LWFA of high-quality beams was recently demonstrated ${ }^{[27,28]}$. In this scheme, using certain initially unmatched laser spot size (the matched condition ${ }^{[12]}$ is defined as $k_{p} w_{0} \approx 2 \sqrt{a_{0}}$, where $k_{p}$ is the plasma wavenumber) and low concentrations of the host gas the ionization-injection condition can be broken due to the self-evolution of the laser pulse and so the wake wave potential, thus shortening the injection length to a few hundred $\mu \mathrm{m}$ of the very-front region of the mixed gas target. This limits the energy spread of the final accelerating electron beam to a percent level. It is worth to mention here that the use of a matched laser spot size for the ionization injection will generate electron beams with continuous spectrum; this has been observed in several experiments $^{[21,23,24,29]}$.

In order to achieve the self-truncated ionization-injection process, two conditions are required to be satisfied at the same time ${ }^{[26]}$. The first one is to use unmatched laser and plasma parameters. In our experiments, we use relatively large laser spot size of $w_{0}=28 \mu \mathrm{m}$, moderate laser power and moderate helium electron density, so we can reach unmatched parameters of $k_{p} w_{0} \sim 11-13$, which is much larger than $2 \sqrt{a_{0}} \sim 1.9-2.5$. The second condition is to use very low doped (nitrogen) gas concentrations, typically less than $1 \%$. Both conditions are satisfied in our experiments. In this paper, we present detailed experimental results and optimization of the self-truncated ionization-injection scheme which leads us to conclude on the robustness of such interesting scheme for the generation of small energy-spread electron beams.

\section{Experiment}

The experiment was conducted using a Ti:sapphire 200 TW laser facility at the Key Laboratory for Laser Plasmas of Shanghai Jiao Tong University in China. Here, we briefly describe the upgrade of the basic experimental configuration introduced in previous papers ${ }^{[30-32]}$.

\subsection{Laser system}

Our laser system provides near-IR pulses with the duration of $30 \mathrm{fs}$, central wavelength of $800 \mathrm{~nm}$, and peak power up to 200 TW. However, in the present experiment, laser pulses with power of $30 \mathrm{TW}$ was used and focused by $f / 20$ off-axis parabolic mirror onto the front edge of a gas jet. The Gaussian $1 / e^{2}$ intensity radius of the laser focus spot was $28 \mu \mathrm{m}$, giving a Rayleigh length $Z_{r}$ of $3.1 \mathrm{~mm}$, and the Strehl ratio of the focus spot was 0.4-0.5. The peak focused laser intensity and the corresponding normalized vector potential, $a_{0}$, were approximately $1.8 \times 10^{18}-5.0 \times$ $10^{18} \mathrm{~W} \mathrm{~cm}^{-2}$ and $0.9-1.5$, respectively. Those laser parameters clearly do not satisfy the bubble regime of the LWFA which is usually conducted at $a_{0}>3$. In the current selftruncated ionization-injection scheme we realize (see below) that laser parameters we are using are typical for ionization injection but not for self-injection experiments ${ }^{[22]}$.

\subsection{Gas target}

The gas target was produced by a 4-mm supersonic slit gas jet which blows a prepared gas mixture of helium and nitrogen. The concentration of the nitrogen gas was in the range of $0.1 \%-1 \%$ relative to the helium concentration. To have an accurate gas mixing ratio, commercially available bottles of industrial standards (mixing error of $0.01 \%$ ) were used and the gas tubes connecting the bottle to the gas jet were evacuated to the level of $\sim 10^{-5}$ mbar before allowing the mixed gases to flow in.

The electron density of the laser-produced pure helium plasma was probed by Nomarski interferometer of $100 \mathrm{fs}$ probe beam in a previous experiment ${ }^{[33]}$. The plasma density measured by interferometry was found to be in agreement with the values obtained using fluid-dynamic calculations provided by the manufacturer ${ }^{[34]}$. It also agreed well with the forward Raman scattering (FRS) density diagnostics performed earlier for an identical gas jet ${ }^{[35]}$. The gas jet nozzle was positioned below the laser spot with a vertical height of $2 \mathrm{~mm}$ and the gas valve was triggered exactly at $4.5 \mathrm{~ms}$ before the arrival of the laser pulse. By varying the stagnation pressure of the gas jet from 2 to 4 atm, we could achieve a helium plasma density $\left(n_{e}\right)$ of $3.3 \times 10^{18}-6.7 \times$ $10^{18} \mathrm{~cm}^{-3}$.

\subsection{Diagnosis of electron beams}

Two DRZ screens $\left(\mathrm{Gd}_{2} \mathrm{O}_{2} \mathrm{~S}\right.$ : Tb x-ray fluorescent screen) are used to detect the electron beams. The first one with an area of $5 \mathrm{~cm} \times 5 \mathrm{~cm}$ was imaged into a 16-bit CCD camera. It was placed in front of a 6-cm-long permanent dipole magnet to diagnose online the spatial profile and pointing angle of the electron beams. The second DRZ screen with a size of $30 \mathrm{~cm}$ (length) $\times 10 \mathrm{~cm}$ (width), was imaged onto an ICCD camera; it is placed after the magnet to diagnose online energy spectrum of the electron beams deflected by the effective magnetic field of $\sim B_{\text {eff }}=0.9 \mathrm{~T}$. The distances from the 

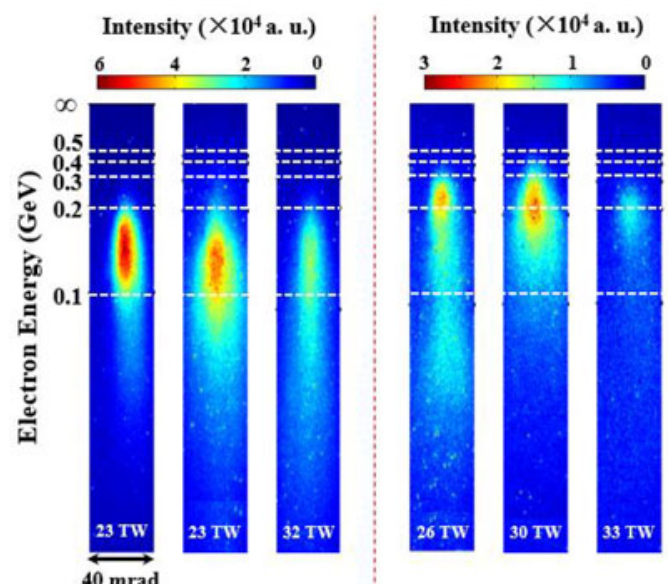

(a) Pure $\mathrm{He}, n_{\mathrm{e}}=4.5 \times 10^{18} \mathrm{~cm}^{-3}$
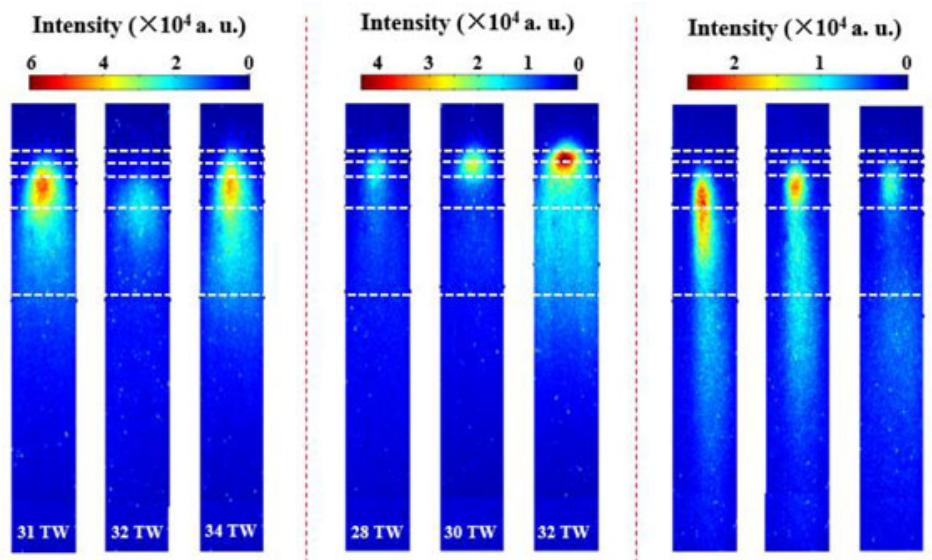

(b) $\mathrm{He}+0.1 \% \mathrm{~N}_{2}, n_{\mathrm{e}}=5 \times 10^{18} \mathrm{~cm}^{-3}:$ (c)

(c) $\mathrm{He}+0.3 \% \mathrm{~N}_{2}, n_{e}=6.7 \times 10^{18} \mathrm{~cm}^{-3}$ (d)

(d) $\mathrm{He}+0.5 \% \mathrm{~N}_{2}, n_{\mathrm{e}}=5 \times 10^{18} \mathrm{~cm}^{-3}$

(e) $\mathrm{He}+1 \% \mathrm{~N}_{2}, n_{\mathrm{e}}=4.2 \times 10^{18} \mathrm{~cm}^{-3}$

Figure 1. Raw images of electron beam energy spectra for 15 shots divided into 5 groups, each group is for a fixed gas mixture concentration. (a) 3 typical spectra for beams generated from laser-driven pure He gas jet, (b) results for $0.1 \% \mathrm{~N}_{2}$ mixed in $99.9 \%$ of $\mathrm{He}$, (c) results for $0.3 \% \mathrm{~N} 2$ mixed in $99.7 \%$ of He, (d) $0.5 \% \mathrm{~N}_{2}$ mixed in $99.5 \%$ of $\mathrm{He}$, and (e) $1 \% \mathrm{~N}_{2}$ mixed in $99 \%$ of He. For (a-e), the unmatched laser-plasma parameter $k_{p} w_{0}$ is $11.2,11.8,13.6,11.8$, and 10.8, respectively. The laser power for all the shots is $30 \mathrm{TW}$ level, and the helium electron density is shown for each group.

gas jet to the first DRZ screen, to the magnet, and to the second DRZ screen were 72,81 , and $161 \mathrm{~cm}$, respectively. A MATLAB electron trajectory code was written to calculate the electron energy shot to shot, taking into consideration the beam's pointing angle before magnet based on the method presented in Ref. [36]. To measure the electron beam energy spread, a computer code was used to deconvolve the energy spectrum from the beam size for each shot. With the $81-\mathrm{cm}$ distance between the magnet entrance and the second DRZ screen, the electron energy resolutions in this experiment are as follows: $1 \%$ at $142 \mathrm{MeV}, 2.1 \%$ at $304 \mathrm{MeV}$, and $2.8 \%$ at $570 \mathrm{MeV}$. A calibrated integrating current transformer (ICT) coupled with a beam charge monitor (BCM) system (Bergoz) was used to measure the accelerated electron beam charge online.

\section{Results and discussions}

\subsection{Experimental results}

Figure 1 shows 5 sets (3 images for each set) of typical raw images for electron beam energy spectra generated from laser-driven wakefield acceleration in gas jets of various concentrations of nitrogen (doped) gas in helium (host) gas from $0 \%$ (pure helium) to $1 \%$ for the unmatched parameters of $k_{p} w_{0}=$ (a) 11.2, (b) 11.8, (c) 13.6, (d) 11.8, and (e) 10.8, respectively. The electron density $n_{e}$ and the peak power of the laser pulses $P$ for each shot are shown on each figure set.

Figure 1(a) shows $\sim 130 \mathrm{MeV}$ (peak energy, not the maximum), board energy-spread ( $40 \%-70 \%$ ) electron beams generated from the pure helium gas jet. For the left and middle shots in this figure, the same laser power (23 TW) was used, generating electron beams with a similar peak energy and relatively low energy spread $(\sim 40 \%)$; for the right shot, the peak energy only gets a little increase but the energy spread drops to $\sim 70 \%$, even at a higher laser power. It is known that it is hard to significantly improve the electron beam energy or energy spread by the self-injection (it works well for matched parameters) in pure helium gas jet for the unmatched parameters we are using. Only by pushing into the matched bubble regime (at matched spot size and higher laser intensities) one may get a higher electron beam quality; however, we are interested in comparing the results of the self-injection with those of the ionization injection for the same unmatched conditions.

Figure 1(b) shows the electron energy spectra obtained from the gas mixture $0.1 \%$ nitrogen $+99.9 \%$ helium. We can see that slightly better quality electron beams are obtained; the peak energies are enhanced to above $\sim 200 \mathrm{MeV}$, and the average energy spread is reduced to $\sim 14 \%$. We can see that more electrons are concentrated in the high-energy part of spectra, which are most likely generated from the ionized nitrogen gas. However, we still see a tail which might come from the self-injected helium electrons which are still dominant in concentration.

By further enhancing the concentration of the nitrogen gas to $0.3 \%$ into $99.7 \%$ He gas, Figure 1(c), the electron beam spectrum quality has been highly enhanced. Now, the mean electron peak energy has increased to $250 \mathrm{MeV}$, and the mean energy spread slightly decreased to $13 \%$. At the same time, the tails of energy spectra have become weaker and shorter.

As the nitrogen gas concentration increased to $0.5 \%$ (mixed with $99.5 \% \mathrm{He}$ ), electron bunch with the highest energy of $454 \mathrm{MeV}$ with an energy spread as low as $3.4 \%$ were observed, as shown in the right panel of Figure 1(d), where almost all electrons are expected to be coming 

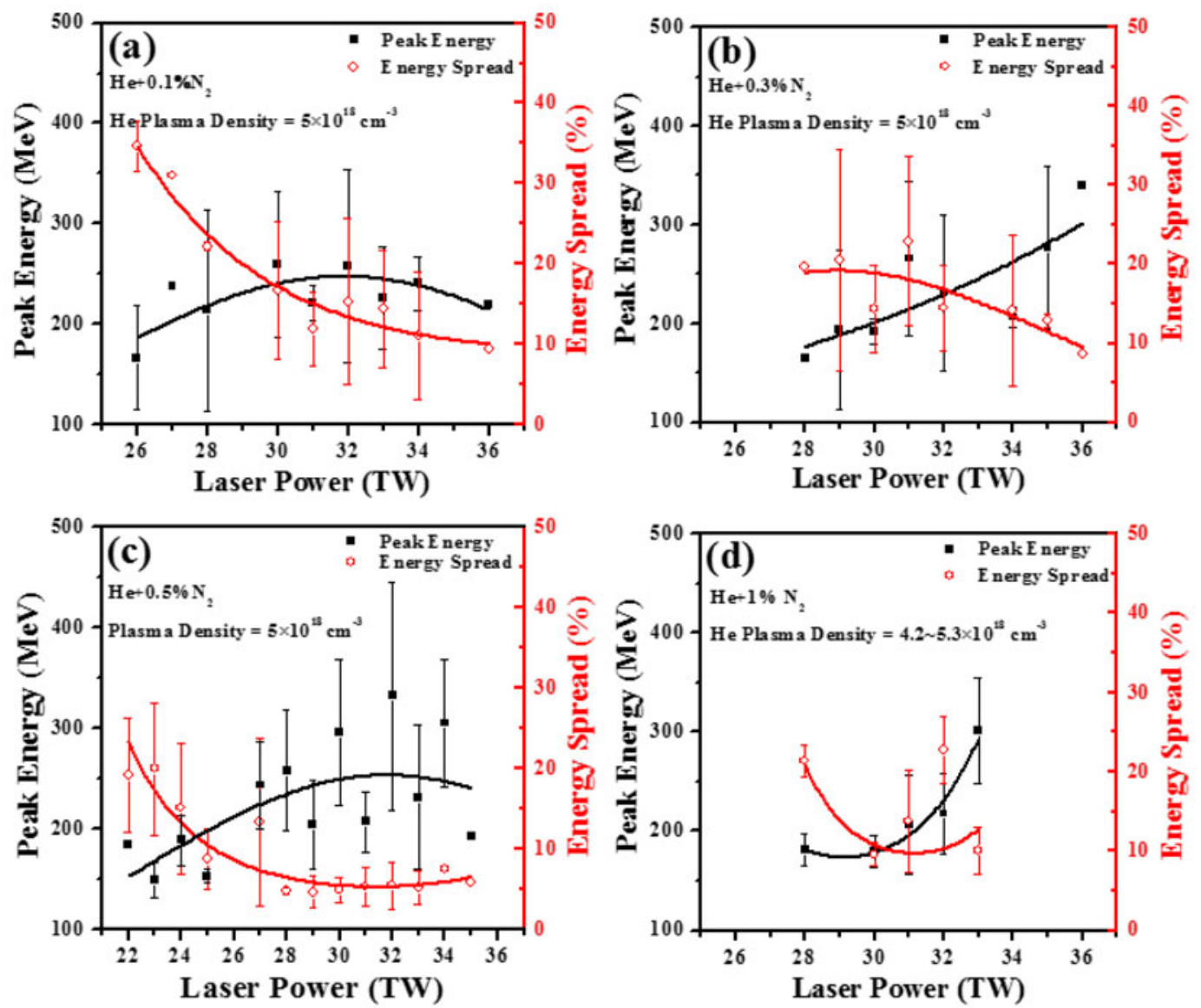

Figure 2. Monoenergetic peak energy and FWHM energy spread of electron beams as a function of laser power for four different concentrations of nitrogenhelium gas mixture targets: (a) $0.1 \% \mathrm{~N}_{2}$ mixed in $99.9 \%$ of $\mathrm{He}$, (b) $0.3 \% \mathrm{~N}_{2}$ mixed in $99.7 \%$ of $\mathrm{He}$, (c) $0.5 \% \mathrm{~N}_{2}$ mixed in $99.5 \%$ of $\mathrm{He}$, and (d) $1 \% \mathrm{~N} 2$ mixed in $99 \%$ of He. The helium plasma density is $5.0 \times 10^{18} \mathrm{~cm}^{-3}$ in all plots, expect for the case of (d) where the density range is slightly different. The unmatched laser-plasma parameters for all points in this graphs are in the range of $k_{p} w_{0} \sim 10.8-12.1$ and $2\left(a_{0}\right)^{1 / 2} \sim 1.9-2.2$.

(due to ionization injection) from the nitrogen's inner shell. Although there is a low-energy tail, it is very weak compared with high-energy part. The other two electron beams in this figure (left and middle panels of Figure 1(d)) are very clean spectra without low-energy electron tails. Their peak energies are 300 and $370 \mathrm{MeV}$, and the energy spreads are $5 \%$ (left) and $5.9 \%$ (middle), respectively.

By further increasing the nitrogen concentration to $1 \%$ (the helium concentration is $99 \%$ ), the situation started to reverse, Figure 1(e), where the electron beam energy dropped to $243 \mathrm{MeV}$ (mean) and the energy spread increased again $(15 \%)$. This case is closer to the case of our previously reported LWFA results in pure nitrogen gas jets which generates long-tail electron beams ${ }^{[32,37]}$. Therefore, we conclude that, given an unmatched laser-plasma parameter, an optimization of the doped gas concentration seems to be very important to achieve an optimum electron beam quality with very small energy spread.

Figure 2 summarizes the experimental results of more shots in support of the results presented in Figure 1; it shows plots for the monoenergetic electron peak energy and energy spread (FWHM) versus the laser power at almost the same plasma density of $5.0 \times 10^{18} \mathrm{~cm}^{-3}$ for the four cases of different concentrations of the doped nitrogen gas. For the case of $1 \%$ nitrogen mixed with $99 \%$ helium, shown in Figure 2(d), we choose the results obtained at a density range of $4.2 \times 10^{18}-5.3 \times 10^{18} \mathrm{~cm}^{-3}$, because of the limited number of useful shots. Firstly, there is a clear trend shown in each plot that as the laser power increases the peak energies of the electron beam increases while the energy spreads decreases. Then, after comparing the four plots, we can find another trend: by gradually increasing the concentration of the nitrogen gas to a certain value $(0.5 \%$ for the above parameters), the beam's monoenergetic energy is significantly enhanced and the reduction of its energy spread are achieved as well; after that, by adding more nitrogen to helium, the quality of electron beams has been degraded.

In Figure 2(a) with the nitrogen ratio of $0.1 \%$, all the average peak energies are below $300 \mathrm{MeV}$; the lowest average energy spread is above or near $10 \%$. By increasing the concentration of $\mathrm{N}_{2}$ to $0.3 \%$ (Figure 2(b)), the highest average peak energy is enhanced to $\sim 300 \mathrm{MeV}$, and the 

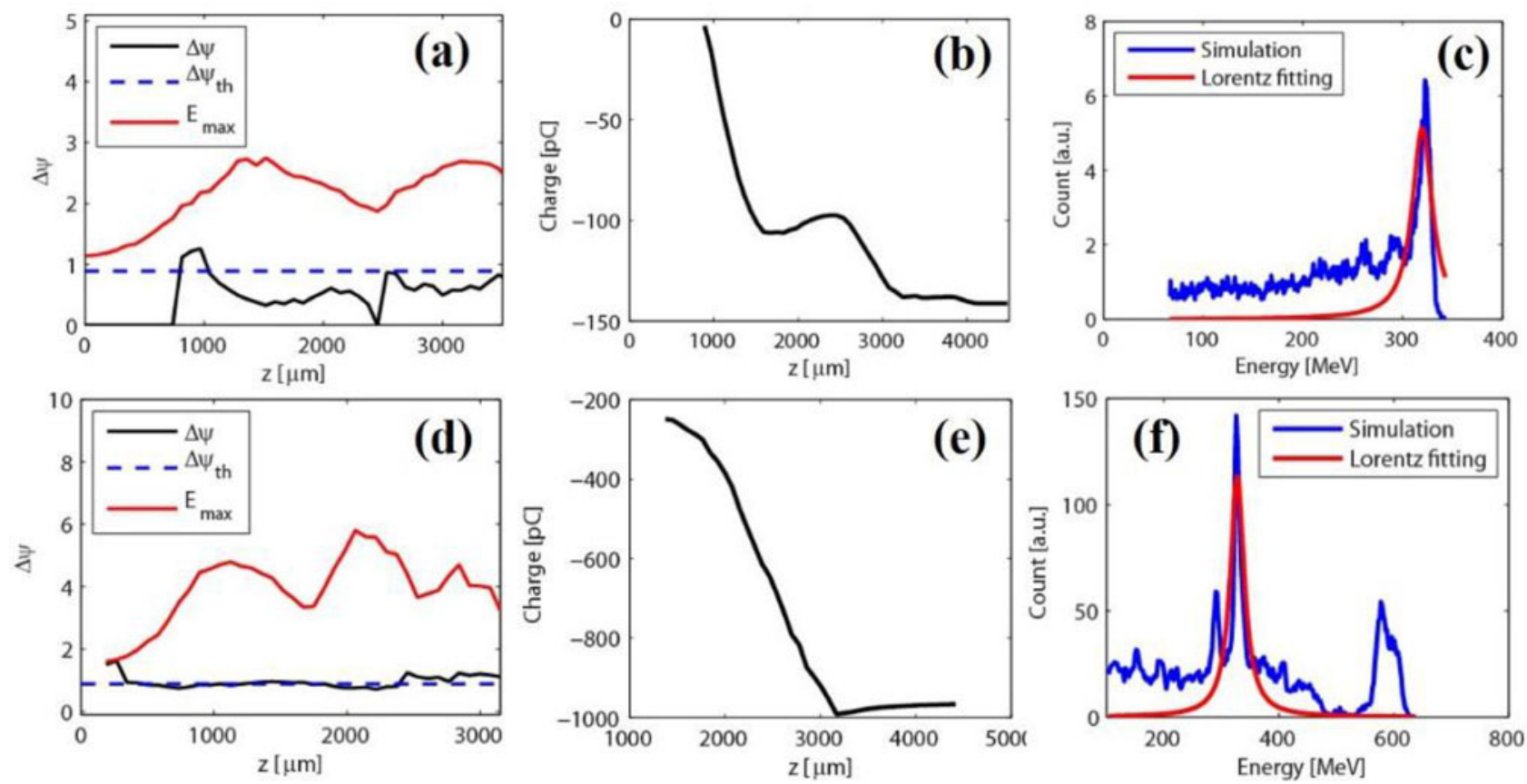

Figure 3. 3D-PIC simulation results using OSIRIS code. Panels (a-c) are results from ionization injection, while (d-f) are from self-injection in pure helium, detailed parameters are shown in the text. (a) and (d) Evolution of the maximum laser electric field and pseudopotential difference; (b) and (e) injected electron charge along the propagation; (c) and (f) electron energy spectra.

lowest average energy spread is reduced to less than $10 \%$. Then the highest average peak energy of $\sim 350 \mathrm{MeV}$ and the lowest average energy spread of $\sim 5 \%$ are observed in Figure 2(c) with the nitrogen concentration of $0.5 \%$. Hereafter, as shown in Figure 2(d), the reverse tendency takes place: the highest average peak energy decreases to $\sim 300 \mathrm{MeV}$ and the lowest average energy spread increases to $\geqslant 10 \%$.

\subsection{D-PIC simulations}

To get more insight on the electron beam acceleration process based on the current version of ionization injection, we carried out 3D-PIC simulations using the code OSIRIS ${ }^{[38]}$ for the laser-plasma conditions close to those used in experiment. In the simulation, an $800 \mathrm{~nm}, 30 \mathrm{fs}, 27 \mathrm{TW}$ laser pulse with initial normalized vector potential $a_{0}=1.14\left(2 \sqrt{a_{0}} \sim\right.$ 2.1 ) is focused at the beginning of the plateau part of the gas mixture with focus radius of $w_{0}=25 \mu \mathrm{m}$. To reduce the simulation time, we only included background plasma (instead of helium) and nitrogen gas. The electron density is $4.4 \times 10^{18} \mathrm{~cm}^{-3}$ and the nitrogen density is $3.4 \times 10^{16} \mathrm{~cm}^{-3}$. The mixture is uniform except for $200 \mu \mathrm{m}$ up-ramp profile in front of the plateau plasma. The number of simulation particles per cell is 4 and total simulation box size is $40 \mu \mathrm{m} \times$ $200 \mu \mathrm{m} \times 200 \mu \mathrm{m}$ with grids number of $1280 \times 200 \times 200$. Simulation time step is $0.104 \mathrm{fs}$. For these parameters, the laser is highly unmatched $\left(k_{p} w_{0}=10 \gg 2 \sqrt{a_{0}} \sim 2.1\right)$ with the plasma and it evolved dramatically.

Figure 3(a) shows evolution of the maximum laser electric field (red curve) and wake pseudopotential difference (black curve) along the laser pulse propagation direction. As we can see, within the front distance of $\sim 800 \mu \mathrm{m}$ the laser intensity gradually increased (due to the self-focusing effect) to the ionization threshold $(E>1.9)$ for the inner-shell electrons of nitrogen $\left(\mathrm{N}^{6+}, \mathrm{N}^{7+}\right)$ which are usually used for ionization injection, where $E$ is the maximum laser's electric field normalized to the factor $m_{e} c \omega_{L} / e$. After that, although the intensity evolves, still it keeps larger than the ionization threshold for a long distance. However, ionization injection could only occur in a very limited region determined by both ionization and wake pseudopotential difference, which is also shown in Figure 3(a). Only when $\Delta \psi>\Delta \psi_{\text {th }} \sim 1$ (blue dashed line), the ionized K-shell electrons can get enough energy from the wakefield to catch up the wake bucket (be trapped by the bucket) before they move to the back of the bucket; otherwise it would just be slipped over by the wake (here, $\Delta \psi=\Delta \psi_{\text {(zioni) }}-\Delta \psi_{\text {(zendbucket) }}$ is the potential difference between the electron ionization position and the end of the first wake wave). The threshold for ionization injection is given by $\Delta \psi_{\mathrm{th}}=1-\gamma_{0}^{-1} \sqrt{\left(1+p^{2} / m_{e}^{2} c^{2}\right)}$. Our case is more universal since the laser intensity evolution not only affects the ionization but also the wake potential itself. From Figure 3(a), we can see that the ionization injection happens in two regions: a major injection at the 
front interaction region $800 \mu \mathrm{m}<z<1000 \mu \mathrm{m}$ and a negligible injection at later position around $z \sim 2600 \mu \mathrm{m}$. The corresponding injected charge evolution is shown in Figure $3(\mathrm{~b})$. About $110 \mathrm{pC}$ of charge is injected into the wakefield in the first injection time and some of them are lost during the following acceleration process. The second (minor) injection gives about $30 \mathrm{pC}$ charge with relatively low energies. In Figure 3(c), we show the final electron beam energy spectrum (at the exit of the plasma medium) by counting only the electrons with energy higher than $50 \mathrm{MeV}$ which are injected during the first injection region. The spectrum width of $5 \%$ and the central energy, $310 \mathrm{MeV}$ are both in reasonable agreement with the experimental results.

Finally, and in order to notice the difference between the self-truncated ionization injection and the usual selfinjection in pure helium plasma, we present a 3D-PIC simulation result shown in Figures 3(d)-3(f) for a pure helium case. In the above ionization-injection simulation, the injection takes place only for the inner-shell nitrogen electrons, while the helium electrons and the outer-shell nitrogen electrons are the oscillating electrons forming the plasma wave; these electrons themselves were untrapped. In the simulation of pure helium we keep the electron density similar to the above case, but we increase the laser power in order to get the self-injection of electrons. In this simulation, the peak power of laser pulse was $47 \mathrm{TW}$ (corresponding to $a_{0}=1.49$ ), the electron density is $5.0 \times 10^{18} \mathrm{~cm}^{-3}$ and the same laser spot size of $28 \mu \mathrm{m}$ then $k_{p} w_{0}=11.9 \gg$ $2 \sqrt{a_{0}} \sim 2.4$; highly unmatched parameters. In Figure 3(d), we can see that the initial laser intensity is high enough to trigger the injection process from the very beginning. Then the laser intensity evolves from $E_{\max } \sim 1.5$ to $E_{\max } \sim 6$ and oscillates about $E_{\max } \sim 4$, while the wake pseudopotential difference remains always equal or slightly higher than unity which is the threshold for injection. The electron injection is continuous in this case as shown in Figure 3(e). The final energy spectrum is shown in Figure 3(f), in which multiple quasimonoenergetic bunches are generated. This is a typical energy spectrum from the self-injection bubble regime scenario of LWFA where multiple bunches are injected due to the evolving bubble (resulting from the laser spot evolution). When the first bunch is injected it gains a maximum energy then it starts dephasing, at that time it combines with a second injected bunch that is accelerating. This leads to increasing the density of electrons in certain energy band leading to the first quasimonoenergetic peak $(\sim 300 \mathrm{MeV})$. The second quasimonoenergetic peak appears as the second injected bunch outruns the first bunch and gains higher energy $600 \mathrm{MeV}$. This phenomenon is clearly very nonlinear $\left(E_{\max } \sim 6\right)$ and is typical in the bubble regime (see Refs. [13-15]) and is different from the robust self-truncated ionization injection demonstrated in this paper.

More simulation runs (not shown in Figure 3) for different laser spot sizes have suggested that there could be a range of optimum spot sizes and laser powers (within the unmatched parameters) for the generation of electron beams with higher quality. Experimental work to verify this possibility may be done in future work. Our present 3D simulations show the main features and characteristics of the self-truncated injection phenomenon.

\section{Conclusion}

In this study, we experimentally demonstrated high-quality electron beams generated in a single-stage LWFA configuration by using self-truncated ionization injection. Under unmatched laser-plasma conditions, the generation of electron beams with peak energy near $500 \mathrm{MeV}$ and few percent energy spread is realized by using optimized mixed gas target (low concentration of nitrogen doped in helium). In contrary to other injection schemes, the STII scheme is straightforward to implement. The robustness of the scheme is manifested in many experimental results presented in Figures 1 and 2. In the near-future work, this concept may be realized for multi-GeV LWFA electron acceleration using higher laser intestines.

\section{Acknowledgements}

The authors thank the OSIRIS Consortium at UCLA and IST for providing access to OSIRIS 2.0 framework. This work was supported by the National '973' Program of China under Grant No. 2013CBA01504. Simulations were supported by Shanghai Supercomputer Center and the center for high performance computing at Shanghai Jiao Tong University.

\section{References}

1. T. Tajima and J. M. Dawson, Phys. Rev. Lett. 43, 267 (1979).

2. E. Esarey, C. B. Schroeder, and W. P. Leemans, Rev. Mod. Phys. 81, 1229 (2009).

3. B. Schroeder, E. Esarey, C. G. R. Geddes, C. Benedetti, and W. P. Leemans, Phys. Rev. ST Accel. Beams 13, 10130 (2010).

4. K. Nakajima, H. Lu, X. Zhao, B. Shen, R. Li, and Z. Xu, Chin. Opt. Lett. 11, 013501 (2013).

5. W. Leemans and E. Esarey, Phys. Today 62, 44 (2009).

6. Z. Huang, Y. Ding, and C. B. Schroeder, Phys. Rev. Lett. 109, 204801 (2012).

7. A. R. Maier, A. Meseck, S. Reiche, C. B. Schroeder, T. Seggebrock, and F. Grüner, Phys. Rev. X 2, 031019 (2012).

8. C. G. R. Geddes, C. Toth, J. V. Tilborg, E. Esarey, C. B. Schroeder, D. Bruhwiler, C. Nieter, J. Cary, and W. P. Leemans, Nature 431, 538 (2004).

9. S. Mangles, C. D. Murphy, Z. Najmudin, A. G. R. Thomas, J. L. Collier, A. E. Dangor, E. J. Divall, P. S. Foster, J. G. Gallacher, C. J. Hooker, D. A. Jaroszynski, A. J. Langley, W. B. Mori, P. A. Norreys, F. S. Tsung, R. Viskup, B. R. Walton, and K. Krushelnick, Nature 431, 535 (2004).

10. J. Faure, Y. Glinec, A. Pukhov, S. Kiselev, S. Gordienko, E. Lefebvre, J. P. Rousseau, F. Burgy, and V. Malka, Nature 431, 541 (2004).

11. A. Pukhov and J. Meyer-Ter-Vehn, Appl. Phys. B 74, 355 (2002). 
12. W. Lu, M. Tzoufras, C. Joshi, F. S. Tsung, W. B. Mori, J. Vieira, R. A. Fonseca, and L. O. Silva, Phys. Rev. ST Accel. Beams 10, 061301 (2007).

13. S. Kalmykov, S. A. Yi, V. Khudik, and G. Shvets, Phys. Rev. Lett. 103, 135004 (2009).

14. I. Kostyukov, E. Nerush, A. Pukhov, and V. Seredov, Phys. Rev. Lett. 103, 175003 (2009).

15. N. Hafz, S. K. Lee, T. M. Jeong, and J. Lee, Nucl. Inst. Meth. Phys. Res. A 637, S51 (2009).

16. W. P. Leemans, B. Nagler, A. J. Gonsalves, C. Tóth, K. Nakamura, C. G. R. Geddes, E. Esarey, C. B. Schroeder, and S. M. Hooker, Nature Phys. 2, 696 (2006).

17. N. A. M. Hafz, T. M. Jeong, I. W. Choi, S. K. Lee, K. H. Pae, V. V. Kulagin, J. H. Sung, T. J. Yu, K. H. Hong, T. Hosokai, J. R. Cary, D. K. Ko, and J. Lee, Nature Photon. 2, 571 (2008).

18. X. Wang, R. Zgadzaj, N. Fazel, Z. Li, S. A. Yi, X. Zhang, W. Henderson, Y. Y. Chang, R. Korzekwa, H. E. Tsai, C. H. Pai, H. Quevedo, G. Dyer, E. Gaul, M. Martinez, A. C. Bernstein, T. Borger, M. Spinks, M. Donovan, V. Khudik, G. Shvets, T. Ditmire, and M. C. Downer, Nature Commun. 4, 1988 (2013).

19. W. P. Leemans, A. J. Gonsalves, H. S. Mao, K. Nakamura, C. Benedetti, C. B. Schroeder, C. Tóth, J. Daniels, D. E. Mittelberger, S. S. Bulanov, J.-L. Vay, C. G. R. Geddes, and E. Esarey, Phys. Rev. Lett. 113, 245002 (2014).

20. D. H. Froula, C. E. Clayston, T. Döppner, K. A. Marsh, C. P. J. Barty, L. Divol, R. A. Fonseca, S. H. Glenzer, C. Joshi, W. Lu, S. F. Martins, P. Michel, W. B. Mori, J. P. Palastro, B. B. Pollock, A. Pak, J. E. Ralph, J. S. Ross, C. W. Siders, L. O. Silva, and T. Wang, Phys. Rev. Lett. 103, 215006 (2009).

21. C. McGuffey, A. G. R. Thomas, W. Schumaker, T. Matsuoka, V. Chvykov, F. J. Dollar, G. Kalintchenko, V. Yanovsky, A. Maksimchuk, and K. Krushelnick, Phys. Rev. Lett. 104, 025004 (2010).

22. M. Chen, E. Esarey, C. B. Schroeder, C. G. R. Geddes, and W. P. and Leemans, Phys. Plasmas 19, 03310 (2012).

23. A. Pak, K. A. Marsh, S. F. Martins, W. Lu, W. B. Mori, and C. Joshi, Phys. Rev. Lett. 104, 025003 (2010).

24. C. E. Clayton, J. E. Ralph, F. Albert, R. A. Fonseca, S. H. Glenzer, C. Joshi, W. Lu, K. A. Marsh, S. F. Martins, W. B. Mori, A. Pak, F. S. Tsung, B. B. Pollock, J. S. Ross, L. O. Silva, and D. H. Froula, Phys. Rev. Lett. 105, 105003 (2010).
25. J. S. Liu, C. Q. Xia, W. T. Wang, H. Y. Lu, C. Wang, A. H. Deng, W. T. Li, H. Zhang, X. Y. Liang, Y. X. Leng, X. M. Lu, C. Wang, J. Z. Wang, K. Nakajima, R. X. Li, and Z. Z. Xu, Phys. Rev. Lett. 107, 035001 (2011).

26. B. B. Pollock, C. E. Clayton, J. E. Ralph, F. Albert, A. Davidson, L. Divol, C. Filip, S. H. Glenzer, K. Herpoldt, W. Lu, K. A. Marsh, J. Meinecke, W. B. Mori, A. Pak, T. C. Rensink, J. S. Ross, J. Shaw, G. R. Tynan, C. Joshi, and D. H. Froula, Phys. Rev. Lett. 107, 045001 (2011).

27. M. Zeng, M. Chen, Z. M. Sheng, W. B. Mori, and J. Zhang, Phys. Plasmas 21, 030701 (2014).

28. S. Li, N. A. M. Hafz, M. Mirzaie, T. Sokollik, M. Zeng, M. Chen, Z. M. Sheng, and J. Zhang, Opt. Express 22, 29578 (2014).

29. C. Kamperidis, V. Dimitriou, S. P. D. Mangles, A. E. Dangor, and Z. Najmudin, Plasma Phys. Control. Fusion 56, 084007 (2014).

30. S. Li, N. A. M. Hafz, M. Mirzaie, X. Ge, T. Sokollik, M. Chen, Z. Sheng, and J. Zhang, J. Appl. Phys. 116, 043109 (2014).

31. S. Li, N. A. M. Hafz, M. Mirzaie, A. M. M. Elsied, X. Ge, F. Liu, T. Sokollik, M. Tao, L. Chen, M. Chen, Z. Sheng, and J. Zhang, Phys. Plasmas 21, 083108 (2014).

32. M. Tao, N. A. M. Hafz, S. Li, M. Mirzaie, A. M. M. Elsied, X. Ge, F. Liu, T. Sokollik, L. Chen, Z. Sheng, and J. Zhang, Phys. Plasmas 21, 073102 (2014).

33. Y. Ma, L. M. Chen, N. A. M. Hafz, D. Z. Li, K. Huang, W. C. Yan, J. Dunn, Z. M. Sheng, and J. Zhang, Appl. Phys. Lett. 105, 161110 (2014).

34. T. Hosokai, K. Kinoshita, T. Watanabe, K. Yoshii, T. Ueda, A. Zhidkov, M. Uesaka, K. Nakajima, M. Kan, and H. Kotaki, in Proceedings of 8th European Particle Accelerator Conference (EPAC) (2002), p. 981.

35. N. M. Hafz, I. W. Choi, J. H. Sung, H. T. Kim, K. H. Hong, T. M. Jeong, and T. J. Yu, Appl. Phys. Lett. 90, 151501 (2007).

36. H. J. Cha, I. W. Choi, H. T. Kim, I. J. Kim, K. H. Nam, T. M. Jeong, and J. Lee, Rev. Sci. Instrum. 83, 063301 (2012).

37. M. Z. Mo, A. Ali, S. Fourmaux, P. Lassonde, J. C. Kieffer, and R. Fedosejevs, Appl. Phys. Lett. 100, 074101 (2012).

38. R. A. Fonseca, L. O. Silva, F. S. Tsung, V. K. Decyk, W. Lu, C. Ren, W. B. Mori, S. Deng, S. Lee, T. Katsouleas, and J. C. Adam, Lect. Notes Comput. Sci. 2331, 342 (2002). 